\title{
Strong interaction between light and a single trapped atom without the need for a cavity
}

\author{
MENG KHOON TEY ${ }^{1}$, ZILONG CHEN ${ }^{2}$, SYED ABDULLAH ALJUNID' ${ }^{1}$, BRENDA CHNG ${ }^{1}$, FLORIAN HUBER ${ }^{3}$, \\ GLEB MASLENNIKOV ${ }^{1}$ AND CHRISTIAN KURTSIEFER ${ }^{1 *}$
}

\author{
${ }^{1}$ Center for Quantum Technologies and Department of Physics, National University of Singapore, 3 Science Drive 2, 117543, Singapore \\ ${ }^{2}$ Institute of Materials Research and Engineering, 3 Research Link, 117602, Singapore \\ ${ }^{3}$ Department of Physics, Technical University of Munich, James Franck Street, 85748 Garching, Germany \\ *e-mail: christian.kurtsiefer@gmail.com
}

Published online: 12 October 2008; doi:10.1038/nphys1096

Many quantum information processing protocols require efficient transfer of quantum information from a flying photon to a stationary quantum system ${ }^{1-3}$. To transfer information, a photon must first be absorbed by the quantum system. This can be achieved, with a probability close to unity, by an atom residing in a high-finesse cavity ${ }^{1}$. However, it is unclear whether a photon can be absorbed effectively by an atom in a free space. Here, we report on an observation of substantial extinction of a light beam by a single ${ }^{87} \mathrm{Rb}$ atom through focusing light to a small spot with a single lens. The measured extinction values can be directly compared to the predictions of existing free-space photon-atom coupling models ${ }^{4-6}$. Our result should open a new perspective on processing quantum information carried by light using atoms, in particular for experiments that require strong absorption of single photons by an atom in free space.

Strong interaction between light and matter is essential for successful operation of many quantum information protocols such as quantum networking ${ }^{1,2}$, entanglement swapping between two distant atoms $s^{3,7,8}$ and implementation of elementary quantum gates ${ }^{9}$. These protocols consider quantum states of localized carriers (nodes), such as atoms, ions or even atomic ensembles, that exchange information through a quantum channel with the help of 'flying' qubits (photons). The quantum channels can be implemented via well-defined photonic modes that couple the nodes with high efficiency. For example, in the original proposal for quantum networks ${ }^{1}$, atoms were placed in high-finesse cavities that not only provide a strong interaction between a photon and an atom, but also ensure that most of the spontaneously emitted photons are collected into the same mode. Experimental advances in atom-photon cavity quantum electrodynamics indeed enabled the information exchange between an atom and single photons in this configuration to be carried out with high efficiency ${ }^{10-14}$. However, scaling such a scheme to many localized nodes is experimentally difficult, because managing the losses and coupling of the intracavity field of high- $Q$ cavities to propagating modes of flying qubits is already quite challenging.

In an attempt to avoid the complications connected with cavities, an interface between stationary and flying qubits in a simpler free-space configuration could be considered, where the

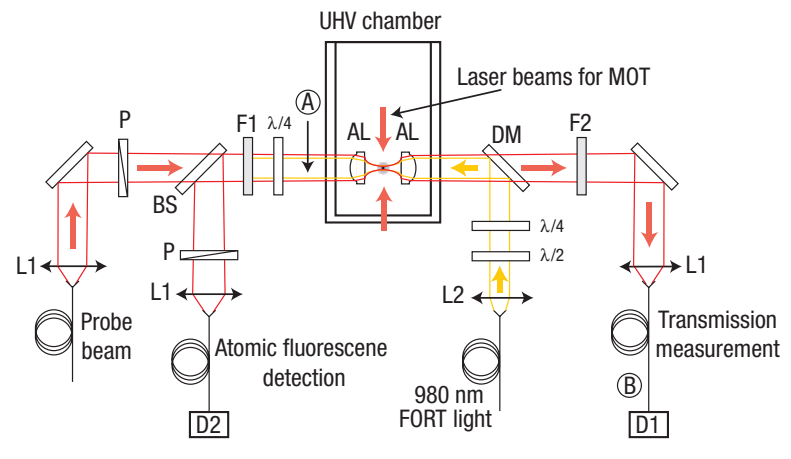

Figure 1 Experimental set-up for measuring the extinction of a light beam by a single atom. AL: aspheric lens $(f=4.5 \mathrm{~mm}$, full $\mathrm{NA}=0.55)$, P: polarizer, $\mathrm{DM}$ : dichroic mirror, BS: beam splitter with $99 \%$ reflectivity, $\lambda / 4, \lambda / 2$ : quarter- and half-wave plates, F1: filters for blocking the $980 \mathrm{~nm}$ FORT light, F2: interference filter centred at $780 \mathrm{~nm}, \mathrm{D} 1$ and D2: Si avalanche photodiodes, UHV: ultrahigh vacuum. Four more laser beams forming the MOT lie in an orthogonal plane and are not shown explicitly.

quantum channel is defined, for example, by a Gaussian mode of a single-mode optical fibre, and a single atom is strongly coupled to this mode with the help of a large-numerical-aperture lens. Indeed, the common model describing the interaction of a monochromatic plane wave with a two-level atom predicts a scattering cross-section of $\sigma=3 \lambda^{2} / 2 \pi$. This area is close to a diffraction-limited spot size of a lens with a large numerical aperture (NA), hence suggesting a high coupling efficiency for such a system. Coupling efficiency here refers to the absorption probability of a flying photon by a stationary quantum system. For a free atom, this absorption probability is equivalent to the scattering probability of photons by the atom. On the other hand, for strong focusing where substantial coupling might be expected, careful consideration of the electric field strength and polarization within the focal 'spot' is necessary $y^{5,15}$ because an atom essentially interacts only with the field at its 


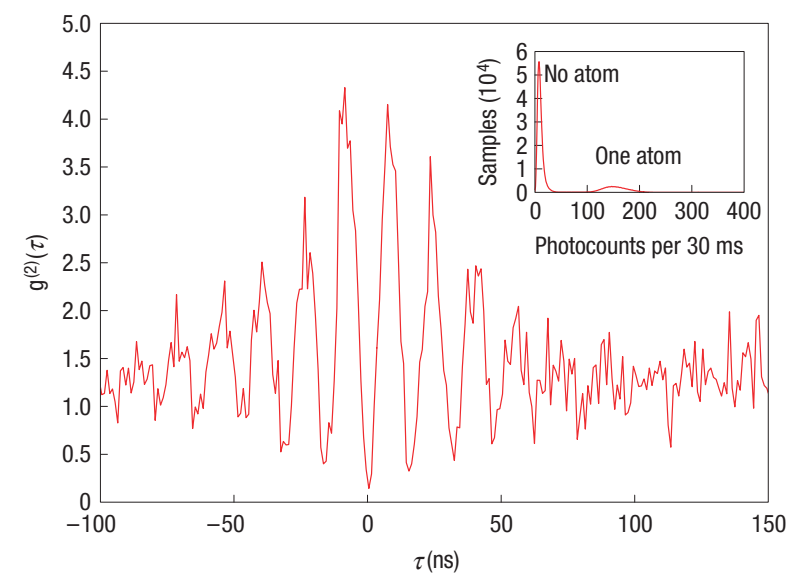

Figure 2 Evidence for single-atom occupancy of our trap. Normalized second-order correlation function versus time delay $\tau$ between two photodetection events at detectors D1 and D2 (not corrected for background counts) with clear antibunching at $\tau=0$. Inset: Histogram of photocounts from the atomic fluorescence revealing the 'binary' character of the detected events due to collisional blockade ${ }^{20}$.

location. The conclusion from such an attempt ${ }^{15}$ was that for realistic lenses, only a low coupling efficiency can be accomplished. In view of these two contradicting opinions, we experimentally quantified the coupling efficiency between a focused light beam and a single atom without the need for a cavity, using a simple transmission measurement set-up.

The first transmission spectrum of a single atom was observed for a ${ }^{198} \mathrm{Hg}^{+}$ion ${ }^{16}$. There, the absorption probability of the probe photons was estimated to be about $2.5 \times 10^{-5}$. Recent experiments on single molecules and semiconductor quantum dots ${ }^{17-19}$ reported a signal contrast up to $13 \%$. The main idea of the experiment presented here is to focus a weak Gaussian light beam with a narrow spectral bandwidth (probe) onto a single ${ }^{87} \mathrm{Rb}$ atom using a single lens. Then, light not scattered by the atom is fully collected by a second lens and delivered to a single-photon detector. Our set-up enables us to directly quantify the scattering (absorption) probability of the probe by a single atom from a measured extinction value (see the Methods section).

Figure 1 shows a schematic diagram of our experiment. The heart of the set-up consists of two identical aspheric lenses (full $\mathrm{NA}=0.55, f=4.5 \mathrm{~mm}$ ), mounted in a confocal arrangement inside an ultrahigh vacuum chamber. The Gaussian probe beam is first delivered from a single-mode fibre, focused by the first lens, fully collected by the second lens and finally coupled again into a single-mode fibre connected to a $\mathrm{Si}$ avalanche photodiode. $\mathrm{A}{ }^{87} \mathrm{Rb}$ atom is trapped at the focus between the two lenses by means of a far-off-resonant optical dipole trap (FORT) formed by a light beam $(\lambda=980 \mathrm{~nm})$ passing through the same lenses. Cold atoms are loaded into the FORT from a magneto-optical trap (MOT) surrounding the FORT with a focal waist of $1.4 \mu \mathrm{m}$ (inferred from the measured input waist using paraxial approximation). For the small size of our FORT, a collisional blockade mechanism allows no more than one atom in the trap at any time $e^{20,21}$. To confirm the single-atom occupancy of the trap, we extract the second-order correlation function $g^{(2)}(\tau)$ from the fluorescence of the trapped atom exposed to the MOT beams (see set-up in Fig. 1). Figure 2 shows $g^{(2)}(\tau)$ measured as a normalized histogram of time delays between photodetection events at detectors D1

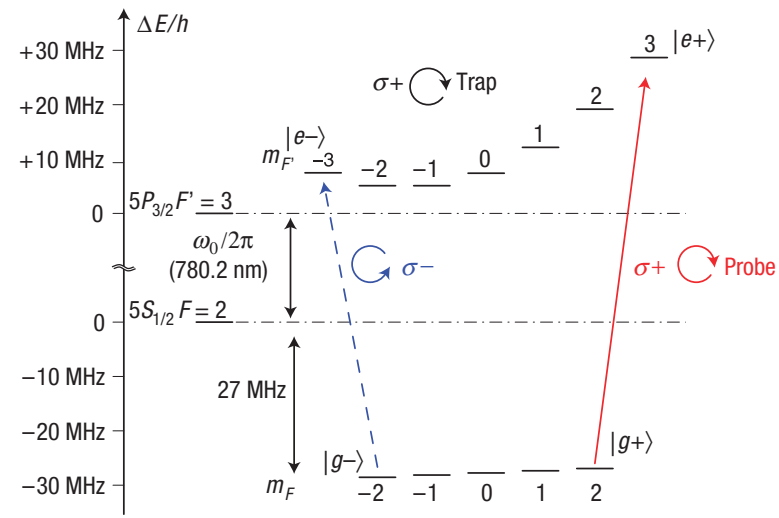

Figure 3 Energy levels and coupling light fields for $\mathrm{a}^{87} \mathrm{Rb}$ atom trapped in a FORT with a circularly polarized trap field. The a.c. Stark shift of the $F=2$ ground states differs only slightly, whereas the (repulsive) shift of the excited states in $F^{\prime}=3$ varies strongly with the magnetic number $m_{F}$. Trap field parameters are chosen to match our experiment.

and D2, which reveals a Rabi oscillation with $\approx 62 \mathrm{MHz}$, damped on a timescale compatible with the spontaneous decay time of the $5 P$ state $(27 \mathrm{~ns})$. An almost vanishing $g^{(2)}(\tau=0)$ indicates that no two photons are emitted at the same time from the trap region, which provides, along with a binary on/off fluorescence signal $^{20}$, strong evidence that we only have a single atom in the trap ${ }^{22-24}$.

The largest extinction would be expected to be observed for a clean two-level system with no other decay channels. Therefore, we use a circularly polarized probe beam that optically pumps the ${ }^{87} \mathrm{Rb}$ atom to a closed-cycling transition either between $|g+\rangle=\left|5 S_{1 / 2}, F=2, m_{F}=+2\right\rangle$ and $|e+\rangle=\left|5 P_{3 / 2}, F^{\prime}=3, m_{F^{\prime}}=+3\right\rangle$, or between $|g-\rangle=\mid F=2$, $\left.m_{F}=-2\right\rangle$ and $|e-\rangle=\left|F^{\prime}=3, m_{F^{\prime}}=-3\right\rangle$ (Fig. 3). A magnetic field orthogonal to the quantization axis causes the atom to undergo Larmor precession, leaking population from $|g \pm\rangle$ or $|e \pm\rangle$ to other $\left|m_{F}\right\rangle,\left|m_{F^{\prime}}\right\rangle$ states, which upsets the clean two-level system. To prevent this, we carefully zero the magnetic field at the location of the trapped atom, and then apply a magnetic bias field along the quantization axis during the measurement. Similarly, the FORT-induced a.c. Stark shift breaks the degeneracy of the hyperfine states of the trapped atom. If $|g \pm\rangle$ and $|e \pm\rangle$ (fixed through optical pumping by the probe) are not energy eigenstates, population also leaks out of the two-level system, resulting in a reduction of the observed extinction by a factor of two for a linearly polarized FORT field. We therefore adopt a circularly polarized FORT beam counterpropagating with the circularly polarized probe.

Figure 3 shows the calculated a.c. Stark shift of the $5 S_{1 / 2}, F=2$ and $5 P_{3 / 2}, F^{\prime}=3$ hyperfine states under the influence of a circularly polarized FORT light of $980 \mathrm{~nm}$ wavelength. The quantization axis of our system is chosen parallel to the main propagation axes of the probe/FORT beams, and such that the polarization of the FORT field is right-hand circular. At the centre of the FORT, the energies of $5 S_{1 / 2}$ states are lowered by an average of $h \cdot 27 \mathrm{MHz}$ (defining the trapping potential) with a small sublevel energy splitting of $\approx 1 \mathrm{MHz}$. The $5 P_{3 / 2}$ levels shift upwards and are strongly split, forming a repulsive potential. The resulting shifts of the resonance frequency for different transitions can be observed directly in a transmission measurement in which the frequency of the probe is tuned over the resonance frequency of the trapped atom. 


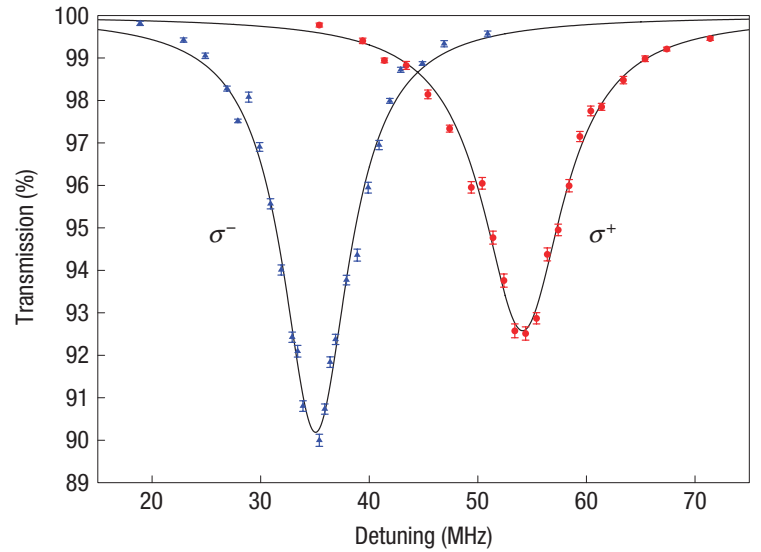

Figure 4 Transmission of the probe beam versus detuning from the natural resonant frequency of the $|g\rangle$ to $|\boldsymbol{e}\rangle$ transition. The absolute photon scattering rate is kept at $\approx 2,500 \mathrm{~s}^{-1}$ for every point by adjusting the probe intensity according to the measured extinction. The solid lines are Lorentzian fits. Error bars indicate \pm 1 standard deviations obtained from propagated Poissonian counting statistics (see the Methods section).

Figure 4 shows the transmission of the probe as a function of detuning from the natural resonant frequency $\omega_{0} / 2 \pi$ of the $|g\rangle$ to $|e\rangle$ transition (see the Methods section for transmission measurement procedures). The two spectra of a single ${ }^{87} \mathrm{Rb}$ atom were obtained for $\sigma^{+}$and $\sigma^{-}$polarized probes, while keeping the handedness of the FORT beam fixed. As expected, the atomic resonance frequency is different for the two probe polarizations, and agrees very well with the prediction shown in Fig. 3. The Lorentzian fit to the transmission spectrum for the $\sigma^{-}$probe shows a maximum extinction of $9.8 \pm 0.2 \%$ with a full-width at half-maximum of $7.5 \pm 0.2 \mathrm{MHz}$. The $\sigma^{+}$probe gives a maximum extinction of $7.4 \pm 0.1 \%$ with a full-width at half-maximum of $9.1 \pm 0.3 \mathrm{MHz}$. As the $\mathrm{D} 2$ transition in ${ }^{87} \mathrm{Rb}$ has a natural linewidth of $6.0 \mathrm{MHz}$ and the linewidth/stability of the probe laser is about $1 \mathrm{MHz}$, we conclude that an atom exposed to the $\sigma^{-}$ probe has been successfully kept in a two-level cycling transition. However, the same conclusion cannot be made for the atom exposed to a $\sigma^{+}$probe. A possible explanation is that optical pumping by the $\sigma^{+}$probe is less effective because a probe frequency resonant to the $|g+\rangle$ to $|e+\rangle$ transition is further detuned from the resonant frequencies of other $\left|F=2, m_{F}\right\rangle$ to $\left|F^{\prime}=3, m_{F^{\prime}}\right\rangle$ transitions, whereas the resonance frequency of $|g-\rangle$ to $|e-\rangle$ is less detuned from other transitions (Fig. 3). Furthermore, a FORT wavelength of $980 \mathrm{~nm}$ forms a repulsive potential for the $5 P_{3 / 2}$ levels. As the energy of $|e+\rangle$ is higher than that of $|e-\rangle$, an atom in $|e+\rangle$ experiences a stronger repulsive force from the FORT. As a result, the atom picks up more kinetic energy under the $\sigma^{+}$probe, resulting in a reduced extinction and line broadening due to the position dependence of the Stark shift in the trap.

Returning to the photon-atom coupling efficiency, we want to emphasize that an extinction of $9.8 \%$ observed for a probe focused to an $\approx 860 \mathrm{~nm}$ waist (again inferred from lens and beam parameters using paraxial optics) is large when compared with results reported from experiments carried out on single molecules and quantum dots ${ }^{17-19}$. There, the excitation light field was either confined with a small aperture of $\approx 100 \mathrm{~nm}$ (ref. 17), or focused by using solid immersion lenses ${ }^{18,19}$ that provide much tighter focusing than in our case. In all of these experiments, quantum systems were embedded into complex solid-state host environments, which complicates the theoretical treatment of light scattering. The conceptual simplicity of the system we investigate and the fact that we directly measure the extinction of the probe beam enables a clean comparison with existing photon-atom coupling models ${ }^{5,6,15}$.

One of the models that closely describes our experiment was presented by van Enk and Kimble ${ }^{15}$. It considers a monochromatic and circularly polarized Gaussian beam focused by an ideal thin lens onto a two-level atomic system. Estimations based on that model gave a very dim outlook on the effectiveness of coupling light to an atom using a lens. In particular, a direct application of the method described there predicts a maximum scattering probability of $2.2 \%$ for our experimental parameters. As it turns out, two approximations adopted in the model (parabolic wave front after the lens, and no change to the polarization of a light beam passing through the lens) greatly underestimated scattering probability for stronger focusing. Dropping these approximations, we find (with otherwise the same methods) a scattering probability of $20.8 \%$ for our experimental parameters ${ }^{25}$. The residual difference between the predicted and measured values could be due both to the imperfections of our aspheric lens, and the fact that the atom is not completely stationary at the focus. Applying this model for an even tighter focus, a very high scattering probability of up to $98 \%$ is predicted (for focusing NA $\approx 0.9)^{25}$. Such a high scattering probability is at odds with other photon-atom coupling models that suggest a maximum scattering probability of $50 \%$ for a light beam focused by a lens as in our set-up ${ }^{5,6}$; further experimental work is required to check this discrepancy.

In the presented coupling scheme, the lens system suffers much less from losses compared with cases where a cavity is used to enhance the coupling of light to an atom ${ }^{13}$. This advantage, together with the simplicity of such a configuration would make such a photon-atom coupling scheme appealing for many applications involving quantum state transfer from photons to atoms. Furthermore, the strong interaction of the atom with a flying qubit suggests using the atom as a mediator for photon-photon interactions, pointing in a new direction for implementing photonic quantum gates.

\section{METHODS}

\section{DIRECT EXTINCTION MEASUREMENTS}

In general, extinction is obtained by comparing the transmitted power of the probe with and without the sample in the optical path of the probe. In usual extinction measurements, for example, as implemented in a commercial spectrophotometer, the probe beam is collected fully by the power-measuring device. However, this is not the case in the extinction measurements on single quantum systems reported so far, for example in refs 17-19. The reason is that substantial extinction of a probe beam by single quantum systems generally requires strong focusing. It is, nevertheless, difficult if not impossible in most experiments to collect the strongly diverging probe fully after the focus.

If we fully collect the excitation mode and connect the measured transmission $T$ to the scattering probability $p_{\mathrm{sc}}$ by $T=1-p_{\mathrm{sc}}+\alpha p_{\mathrm{sc}}$, where $\alpha$ represents the percentage of scattered light collected by the transmitted power detector, then the extinction $\epsilon=1-T$ is related to the scattering probability by $p_{\mathrm{sc}}=\epsilon /(1-\alpha)$. The collection efficiency $\alpha$ in this experiment is estimated to be less than $5 \%$, so $p_{\mathrm{sc}} \approx \epsilon$.

\section{LOSSES AND INTERFERENCE ARTEFACTS}

We carefully quantified the losses in the transmission channel to make sure our results do not suffer from interference artefacts in the sense that interference between a partially collected probe and scattered light can lead to an extinction larger than the scattering probability. The total transmission from point $\mathrm{A}$ in Fig. 1 (before the vacuum chamber) to point B (after the single-mode fibre and just before the detector) is $53 \%$. The $47 \%$ loss includes $21.6 \%$ loss from the four uncoated window surfaces of the vacuum chamber and the two aspheric lenses; $5.3 \%$ loss over two dichroic mirrors, an interference filter (peak transmission $T=96 \%$ at $780 \mathrm{~nm}$ ) and a mirror; and $28.4 \%$ coupling loss into an uncoated 
single-mode fibre. All of the losses are caused by reflection except for $20 \%$ loss at the fibre coupling that is due to imperfect mode matching. As the scattered field and the probe field should experience the same reflection loss at each surface, we are reasonably confident that our results are free from interference artefacts.

\section{SEQUENCE FOR TRANSMISSION MEASUREMENT}

Once an atom is loaded into the FORT, it triggers the transmission measurement sequence. The main steps include: (1) switching off the MOT beams and the MOT quadrupole coil current; (2) application of a magnetic bias field of $\approx 2 \mathrm{G}$ along the quantization axis; (3) waiting for $20 \mathrm{~ms}$ so that current in the coils stabilizes and optically pumping the atom into either $|g+\rangle$ or $|g-\rangle$ at the same time; (4) recording the photocounts $n_{m}$ of the transmitted probe beam for $\tau_{m}$ ranging from 130 to $140 \mathrm{~ms}$ with detector D1; (5) switching on the MOT beams to check whether the atom is still in the FORT by monitoring fluorescence with detector D2; if 'yes', turn off the MOT beams and repeat steps 3 and 4; (6) otherwise, recording the photocounts $n_{r}$ of the transmitted probe beam with detector D1 for $\tau_{r}=2 \mathrm{~s}$ without an atom in the trap for reference; (7) turning on the MOT beams and the quadrupole coil current, waiting for another atom to be loaded in the FORT.

A transmission value $T$ is obtained for each atom trapping event by $T=\left(\sum n_{m} / \sum \tau_{m}\right)\left(\tau_{r} / n_{r}\right)$, where the summation is carried over all contiguous measurement intervals $m$ for which an atom was found in the trap. The average time an atom stays in the FORT is about $1.5 \mathrm{~s}$. A single data point in Fig. 4 is the average of about 100 of such transmission values, each weighted by $\tau_{r} \sum \tau_{m} /\left(\tau_{r}+\sum \tau_{m}\right)$.

As the MOT beams are turned off during the measurement, the atom can be heated up and even kicked out of the FORT by the probe. To avoid this problem, the intensity of the probe for each detuning is adjusted to result in an actual photon scattering rate of 2,500 s$~^{-1}$ (about five times smaller than the longitudinal oscillation frequency of the atom in the FORT).

The error in the measurements shown in Fig. 4 is dominated by photocounting shot noise. During the transmission measurement process, the atom may fall into the $\left|5 S_{1 / 2}, F=1\right\rangle$ metastable ground state, which is off-resonant with the probe. To bring it back to the pumping cycle, circularly polarized light resonant with the D1 transition is mixed into the probe beam, and later removed with an interference filter F2 (Fig. 1).

Received 27 March 2008; accepted 4 September 2008; published 12 October 2008.

\section{References}

1. Cirac, J. I., Zoller, P., Kimble, H. J. \& Mabuchi, H. Quantum state transfer and entanglement distribution among distant nodes in a quantum network. Phys. Rev. Lett. 78, 3221-3224 (1997).

2. Duan, L.-M., Lukin, M. D., Cirac, J. I. \& Zoller, P. Long-distance quantum communication with atomic ensembles and linear optics. Nature 414, 413-418 (2001).

3. Blinov, B. B., Moehring, D. L., Duan, L.-M. \& Monroe, C. Observation of entanglement between a single trapped atom and a single photon. Nature 428, 153-157 (2004).
4. Cohen-Tannoudji, C., Grynberg, G. \& Dupont-Roc, J. Atom-Photon Interactions: Basic Processes and Application (Wiley, 1992).

5. van Enk, S. J. Atoms, dipole waves, and strongly focused light beams. Phys. Rev. A 69, 043813 (2004)

6. Sondermann, M. et al. Design of a mode converter for efficient light-atom coupling in free space. Appl. Phys. B 89, 489-492 (2007).

7. Matsukevich, D. N., Maunz, P., Moehring, D. L., Olmschenk, S. \& Monroe, C. Bell inequality violation with two remote atomic qubits. Phys. Rev. Lett. 100, 150404 (2008).

8. Volz, J. et al. Observation of entanglement of a single photon with a trapped atom. Phys. Rev. Lett. 96, 030404 (2006).

9. Savage, C. M., Braunstein, S. L. \& Walls, D. F. Macroscopic quantum superpositions by means of single-atom dispersion. Opt. Lett. 15, 628-630 (1990).

10. Meschede, D., Walther, H. \& Müller, G. One-atom maser. Phys. Rev. Lett. 54, 551-554 (1985).

11. Turchette, Q. A., Hood, C. J., Lange, W., Mabuchi, H. \& Kimble, H. J. Measurement of conditional phase shifts for quantum logic. Phys. Rev. Lett. 75, 4710-4713 (1995).

12. Pinkse, P. W. H., Fischer, T., Maunz, P. \& Rempe, G. Trapping an atom with single photons. Nature 404, 365-368 (2000).

13. Boozer, A. D., Boca, A., Miller, R., Northup, T. E. \& Kimble, H. J. Reversible state transfer between light and a single trapped atom. Phys. Rev. Lett. 98, 193601 (2007).

14. Gleyzes, S. et al. Quantum jumps of light recording the birth and death of a photon in a cavity. Nature 446, 297-300 (2007).

15. van Enk, S. J. \& Kimble, H. J. Strongly focused light beams interacting with single atoms in free space. Phys. Rev. A 63, 023809 (2001)

16. Wineland, D. J., Itano, W. M. \& Bergquist, J. C. Absorption spectroscopy at the limit: Detection of a single atom. Opt. Lett. 12, 389-391 (1987).

17. Gerhardt, I. et al. Strong extinction of a laser beam by a single molecule. Phys. Rev. Lett. 98 033601 (2007).

18. Vamivakas, A. et al. Strong extinction of a far-field laser beam by a single quantum dot. Nano Lett. 7, 2892-2896 (2007)

19. Wrigge, G., Gerhardt, I., Hwang, J., Zumofen, G. \& Sandoghdar, V. Efficient coupling of photons to a single molecule and the observation of its resonance fluorescence. Nature Phys. 4, 60-66 (2008).

20. Schlosser, N., Reymond, G., Protsenko, I. \& Grangier, P. Sub-Poissonian loading of single atoms in a microscopic dipole trap. Nature 411, 1024-1027 (2001).

21. Schlosser, N., Reymond, G. \& Grangier, P. Collisional blockade in microscopic optical dipole traps. Phys. Rev. Lett. 89, 203005 (2002).

22. Diedrich, F. \& Walther, H. Nonclassical radiation of a single stored ion. Phys. Rev. Lett. 58 203-206 (1987).

23. Weber, M., Volz, J., Saucke, K., Kurtsiefer, C. \& Weinfurter, H. Analysis of a single-atom dipole trap. Phys. Rev. A 73, 043406 (2006).

24. Gomer, V. et al. Decoding the dynamics of a single trapped atom from photon correlations. Appl. Phys. B 67, 689-697 (1998).

25. Tey, M. K. et al. Interfacing light and single atoms with a lens. Preprint at $<$ http://arxiv.org/abs/0804.4861> (2008)

\section{Acknowledgements}

We would like to acknowledge helpful discussions with V. Scarani, A. Lamas-Linares and H. Loh. This work was partially supported by the Singapore Ministry of Education under FRC grant R-144-000-174-112. Z.C. acknowledges support from the Agency for Science, Technology and Research, Singapore (A*STAR).

\section{Author contributions}

Project planning and data analysis: M.K.T., G.M. and C.K.; experimental work: all authors; numerical work on focusing: M.K.T. and F.H.

Author information

Reprints and permissions information is available online at http://npg.nature.com/reprintsandpermission Correspondence and requests for materials should be addressed to C.K. 